Trauma Berufskrankh 2009 · 11 [Suppl 2]: 203-206

DOI 10.1007/s10039-008-1444-3

Online publiziert: 3. Oktober 2008

(c) Springer Medizin Verlag 2008
U.-J. Gerlach · C. Grimme · R. Schoop
Septische Knochen- und Gelenkchirurgie, BG-Unfallkrankenhaus, Hamburg

\section{Akute posttraumatische Osteitis}

\section{Ist die Osteosynthese zu retten?}

Die akute Osteitis ist eine schwerwiegende Komplikation der Unfallchirurgie und Orthopädie. Es handelt sich um eine bakterielle Infektion oder Besiedelung nach offenen Brüchen oder nach operativer Versorgung von Knochenbrüchen.

\section{Inzidenz}

Die Häufigkeit der akuten Osteitis wird in der Literatur

- für Elektiveingriffe mit 0,1-1,7\%,

- für die Versorgung von geschlossenen Frakturen mit 1-8,1\% [2, 11] sowie

- für die Versorgung von offenen Frakturen verschiedener Schweregrade mit $2,7-43 \%[3,7,9]$

angegeben. Es ist von mindestens 1000 neuen Erkrankungsfällen im Jahr auszugehen [8], bei zunehmender Alterung der Bevölkerung und damit zunehmenden Komorbiditäten ist die Zahl weitaus höher anzusetzen.

\section{Definitionen}

Eine Knocheninfektion innerhalb der ersten 4 Wochen nach einem Trauma oder operativer Versorgung ist als akute Osteitis zu klassifizieren. Knochenentzündungen, die nach 4 Wochen auftreten, werden als chronische Osteitis bezeichnet. Diese Unterscheidung ist uneinheitlich, bei den Infektionen der Endoprothesen wird die Grenze ebenfalls 4 Wochen postoperativ gezogen [13]. Zu diesem Zeitpunkt ist die Biofilmbildung noch nicht abgeschlossen.

\section{Pathogenese}

Die Entstehung der posttraumatischen und postoperativen akuten Osteitis wird maßgeblich durch die Anzahl und die Virulenz der Erreger sowie das Ausmaß des Wirtsschadens bestimmt. Entscheidend sind die lokale Keimkontamination und eine Minderung der lokalen und systemischen Immunabwehr. Wenn die Keimeinschleppung ausreichend massiv und die Infektabwehr ausreichend gestört sind, kommt es zum Zusammenbruch der Infektabwehr und zur rapiden Keimvermehrung.

\section{Ursachen}

Eine massive Keiminokulation kann durch das Trauma selbst entstehen, z. B. bei offenen Frakturen mit oder ohne begleitenden Gewebeverlust. Der Weichteilund Knochenschaden und die damit verbundene Störung der Durchblutung von Weichteilen und Knochen stellen einen günstigen Nährboden durch nekrotische Weichteile, herausgelöste Fragmente und Hämatome für die Ansiedelung und Vermehrung von Keimen dar. Durch die beschriebene Schädigung kann die Infektion leicht auf Periost, Kortikalis und Markraum übergreifen.

Weitere, die Ausbildungen einer akuten Infektion begünstigende exogene Faktoren sind eine falsche Operationsindikation, eine traumatisierende Operationstechnik, der falsch gewählte Operationszeitpunkt nach dem Trauma und ein möglicherweise falsch gewähltes Implantat.

Die lokale und systemische Immunkompetenz ist durch Risikofaktoren wie Lebensalter über 65 Jahre, Diabetes mellitus, Durchblutungsstörungen, Adipositas, Nikotin- und Alkoholabusus und Herz-Kreislauf-Erkrankungen reduziert. Weiterhin tritt eine akute posttraumatische Osteitis vermehrt bei Patienten mit Immunschwächen oder Medikation mit Immunsuppressiva sowie bei Patienten mit Erkrankungen aus dem rheu-
Abb. 1 Klinisches Bild einer akuten Osteitis nach minimalinvasiv durchgeführter Osteosynthese

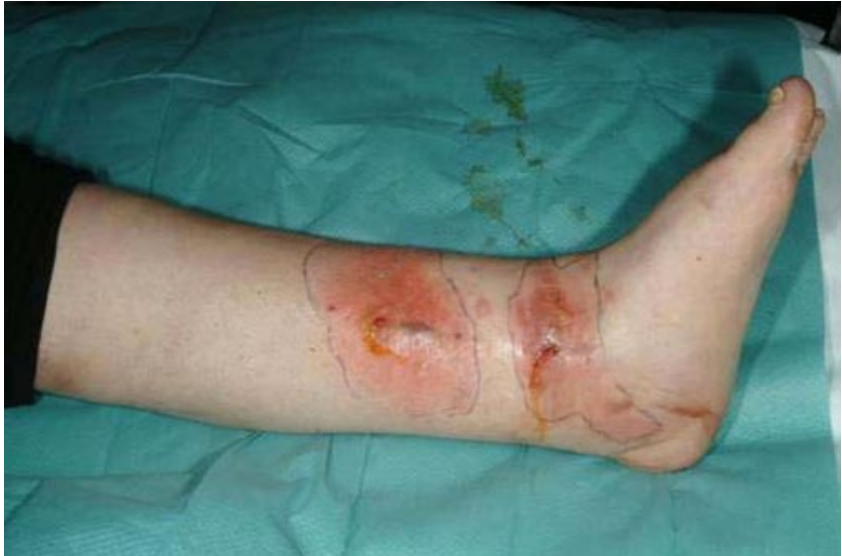




\section{Behandlungsalgorithmen}
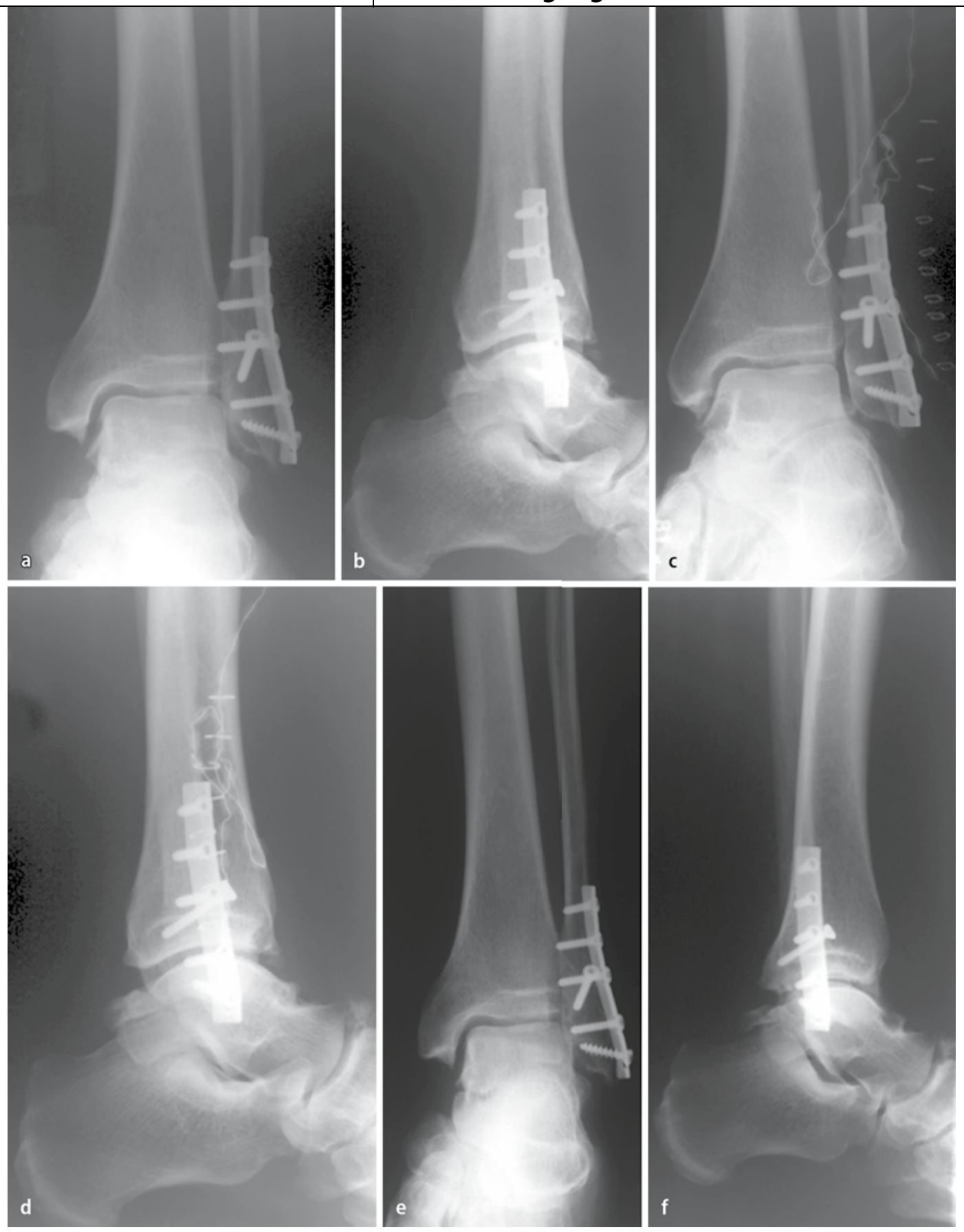

Abb. $2 \varangle$ Außenknöchelfraktur, a,b Röntgenbild nach primärer Versorgung bei klinisch akuter Osteitis, c,d Röntgenkontrolle nach Débridement des Plattenlagers, Arthrotomie, Reimplantation der Osteosyntheseplatte und Septopal ${ }^{\circledR}$-Einlage, e,f Röntgenbild nach Septopal ${ }^{\oplus}$-Entfernung, knöcherner Durchbau der Fraktur bei dauerhafter Infektberuhigung

matischen Formenkreis, mit konsumierenden Erkrankungen oder mit Strahlenschäden auf.

Die lokale Immunkompetenz des Körpers ist durch die Anwesenheit von Implantaten herabgesetzt, nach deren Einbringen steigt die Gefahr der Keimbesiedlung und der resultierenden Infektionen. Implantatfreie Knochen benötigen $100 \times 10^{8}$ Keime zur Entstehung einer Infektion, bei Knochen mit Implantaten reichen $10^{5}$ Keime aus [15]. Durch das einliegende Implantat kommt es zu einer Re- duktion der Phagozytosefähigkeit der körpereigenen Abwehrzellen.

\section{Besiedelung durch Bakterien}

Bei einer Keimbesiedlung binden sich die Bakterien über Adhäsine an das Implantat. Sie bilden dort durch einen bis zu $40 \mu \mathrm{m}$ dicken Biofilm $[4,6,10]$ gesicherte Kolonien. Der Biofilm bietet Schutz vor Antikörpern und Phagozytose. Anfänglich erfolgt die Besiedelung in einer planktonischen Form, d. h. es liegen eine hohe
Stoffwechselrate und eine rasche Vermehrung der Bakterien vor. Die Abwehrreaktion des Körpers wird ausgelöst, es entstehen die typischen klinischen Symptome. In dieser Phase sind die Keime gegenüber Antibiotika sensibel.

In der sessilen Phase verlangsamen sich die biologischen Reaktionen, die Reproduktionsrate ist deutlich reduziert. Durch den Schutz des gebildeten Biofilms sind die Keime teilweise um den Faktor 1000 unempfindlicher gegenüber Antibiotika. Unter dem Schutz des Biofilm kön- 
nen die Bakterien Informationen wie Resistenzen austauschen und weitergeben $[5,12]$. In dieser Phase entziehen sich die Keime der körpereigenen Abwehr.

Ein bakteriell besiedeltes Implantat kann nach der Biofilmbildung nicht mehr in einen keimfreien Zustand gebracht werden, ein Erhalt der Osteosynthese ist dann nicht mehr möglich. Aufgrund der dargestellten Pathophysiologie ist die Grenze zwischen akuter und chronischer Knocheninfektion bei 4 Wochen festzulegen.

\section{Diagnose}

Bei der Diagnostik der akuten posttraumatischen Osteitis steht der klinische Befund im Vordergrund. Klinische Leitsymptome der akuten sind im Gegensatz zur chronischen Osteitis die klassischen Infektzeichen (Rubor, Calor, Dolor, Functio laesa, 0 Abb. 1). Laborchemisch deuten der fehlende Rückgang oder der erneute Anstieg der Entzündungsparameter (Leukozyten, Blutsenkungsgeschwindigkeit, C-reaktives Protein) auf eine frühe Infektion hin. Bildgebende Verfahren wie Röntgen, MRT und die 3-Phasen-Skelettszintigraphie bringen keine weitere Information und sind bei der Diagnosestellung der akuten posttraumatischen Osteitis nicht zielführend. So zeigt die konventionelle Röntgenaufnahme frühestens nach 3-4 Wochen periostale Reaktionen, Osteolysen oder Resorptionszonen. Bei der Diagnosestellung handelt es sich somit um eine rein klinische Diagnose. Die geeignete Vordiagnostik ist klinisch die regelmäßige Wundkontrolle nach operativer Versorgung.

Bei Verdacht auf einer Frühinfektion ist eine Diagnose zu erzwingen. Der klinische Verdacht auf eine Frühinfektion nach Osteosynthese stellt eine dringliche, nicht aufschiebbare Indikation zur Revisionsoperation da. Die notwendige radikale chirurgische Revision hat frühzeitig zu erfolgen.

Ein fehlender Keimnachweis bei möglicherweise vorliegendem Defekt oder Sekretion aus einer Wunde schließt eine Frühinfektion nicht aus, da eine solche aufgrund einer vorausgehenden Antibiotikatherapie trotz negativem Keimnachweises vorliegen kann.
Die Entnahme von Material zur bakteriologischen Untersuchung hat korrekt zu erfolgen, Abwisch- oder Abtupfpräparate erbringen seltener einen Keimnachweis als Gewebeproben. Es ist eine Gewebeprobe oder ein Abradat einzusenden [ 1 , $14,16]$. Es sollten keine bakteriologischen Abstriche aus Eiter genommen werden, da dieser häufig steril ist.

\section{Therapie}

Entscheidend bei der Behandlung der akuten Osteitis ist die frühzeitige radikale chirurgische Revision. Behandlungsziel ist die dauerhafte Infektberuhigung des Knochens und des häufig mitbeteiligten, den Knochen umgebenden Weichteilgewebes bei Stabilität.

Das operative Vorgehen ist abhängig vom Befund, der Lokalisation der Infektion, der Stabilität des Knochens und den individuellen Begleiterkrankungen.

Die alte Narbe sollte eröffnet und als operativer Zugang verwendet werden. Eine ggf. bestehende Fistel ist auszuschneiden. Ein Débridement der umgebenden, häufig mitbeteiligten Hautweichteile ist unerlässlich. Sämtliche entzündlich veränderten Weichteilgewebeanteile werden entfernt. Bei minimalinvasiv eingebrachten Implantaten müssen auch die zwischen den beiden Inzisionen gelegene Hautbrücke eröffnet und das darunter liegende Gewebe radikal débridiert werden.

Bei Stabilität sollte versucht werden, die Osteosynthese zu erhalten. Das Plattenlager muss in diesem Fall gründlich gesäubert werden. Häufig ist es dazu notwendig, die Platte zu lösen und das knöcherne Plattenlager zu débridieren. Bei avitalen Fragmenten unter der einliegenden Osteosyntheseplatte infolge gestörter Durchblutung kann ein ungestörter Heilverlauf mit dauerhafter Infektberuhigung nicht erwartet werden, deswegen müssen diese Knochenanteile radikal entfernt werden. Auf den Knochen kann ein Antibiotikum abgebendes Vlies (Gentacoll $^{\circledR}$, Septocoll ${ }^{\circledR}$ ) aufgelegt werden. Die Osteosyntheseplatte wird anschließend reimplantiert, sodass das Antibiotikavlies zwischen Knochen und Platte liegt.

Bei gelenknahen Frakturen, z. B. Außenknöchelfraktur, sollte immer eine Arthrotomie des angrenzenden Gelenks er-
Trauma Berufskrankh 2009 - 11 [Suppl 2]:

203-206

DOI 10.1007/s10039-008-1444-3

(c) Springer Medizin Verlag 2008

\section{U.-J. Gerlach · C. Grimme · R. Schoop \\ Akute posttraumatische Osteitis. Ist die Osteosynthese zu retten?}

\section{Zusammenfassung}

Die akute Osteitis ist eine schwerwiegende Komplikation der Unfallchirurgie und Orthopädie. Es handelt sich um eine bakterielle Infektion oder Besiedelung des Knochens und des umgebenden Weichteilgewebes nach offenen Brüchen oder nach operativer Versorgung von Knochenbrüchen. Die Diagnose wird klinisch gestellt. Die radikale chirurgische Revision hat frühzeitig zu erfolgen. In vielen Fällen ist der Erhalt des Osteosynthesematerials möglich, jedoch nicht bei Instabilität oder Frühinfekt bei einliegendem Marknagel.

\section{Schlüsselwörter}

Akute Osteitis · Biofilm · Radikale chirurgische Revision · Lokaler Antibiotikumträger .

Sequestrektomie

\section{Acute posttraumatic osteomyelitis. Is it possible to save the osteosynthesis?}

\section{Abstract}

Acute osteomyelitis, a bacterial infection of the bone and the surrounding soft tissue after an open fracture or surgical treatment, is a severe complication in orthopaedic and trauma surgery. If not treated correctly, it is catastrophic for the patient. The diagnosis is a clinical one. Radical surgery must be performed as early as possible. In most cases it is possible to save the osteosynthesis, but not if a nail is infected or if the infection is combined with instability.

\section{Keywords}

Acute osteomyelitis · Biofilm · Radical surgery · Local antibiotic carrier - Sequestrectomy 
folgen. Nach Revision desselben und Ausschluss eines akuten Empyems müssen eine Lavage des Gelenks erfolgen und ein lokaler Antibiotikumträger (s. oben) intraartikulär eingelegt werden (• Abb. 2).

Ist aufgrund von Instabilität, ausgeprägten Infektionen, ausgedehnten begleitenden Hautweichteildefekten oder bekannter Begleiterkrankung der Erhalt der Ostesynthese nicht möglich, muss das einliegende Osteosynthesematerial entfernt werden. Die Stabilisierung hat dann durch einen Fixateur externe zu erfolgen. Lokal ist eine radikale Sequestrektomie notwendig, anschließend wird ein Antibiotikumträger (Septopal ${ }^{\triangleright}$, Gentacoll ${ }^{\oplus}$, Septocoll $^{\circledast}$ ) eingelegt.

Frühinfektionen nach Marknagelosteosynthesen erlauben keinen Erhaltungsversuch. Die Wunden sind zu eröffnen und zu débridieren, die einliegenden Marknägel müssen explantiert werden. Nach Markraumaufbohrung und Einlage von lokalen Antibiotikumträgern in denselben muss die Fraktur über einen Fixateur externe stabilisiert werden.

Sowohl beim Erhaltungsversuch als auch bei der Explantation des Osteosynthesematerials ist postoperativ eine kurzzeitige systemische Antibiose (7-10 Tage) durchzuführen.

Bestehen bei akuten posttraumatischen Osteitiden größere Hautweichteildefekte, sind diese in einem 2. operativen Schritt zu decken, entweder durch lokale plastische Maßnahmen oder durch freie Lappenplastiken. Die endgültige Weichteildeckung hat frühzeitig und stabil zu erfolgen.

Ein möglicherweise resultierender Knochendefekt nach radikaler Sequestrektomie wird nach Infektberuhigung durch Spongiosaplastik oder, bei zirkulären Defekt über $3 \mathrm{~cm}$, durch Segmenttransport aufgebaut.

Parallel zu den oben genannten Maßnahmen wird ein umfassendes Rehabilitationsprogramm mit Physio-, Ergo-, Sportsowie Gehschultherapie durchgeführt.

\section{Korrespondenzadresse}

\section{Dr. U.-J. Gerlach}

Septische Knochen- und Gelenkchirurgie,

BG-Unfallkrankenhaus Hamburg,

Bergedorfer Straße 10, 21033 Hamburg

U.J.Gerlach@buk-hamburg.de
Interessenkonflikt. Der korrespondierende Autor gibt an, dass kein Interessenkonflikt besteht.

\section{Literatur}

1. Bauer TW, Parvizi J, Kobayashi N et al. (2006) Diagnosis of periprosthetic infection. J Bone Joint Surg Am 88: 869-882

2. Bhandari M, Guyatt GH, Tond D et al. (2000) Reamed versus unreamed intramedullary nailing of lower extremity long bone fractures: a systematic overview and metaanalysis. J Orthop Trauma 14: 2-9

3. Carsenti-Etesse H, Doyon F, Desplaces N et al. (1999) Epidemiology of bacterial infection during management of open leg fractures. Eur J Clin Microbiol Infect Dis 18: 315-323

4. Donlan RM (2002) Biofilms: microbial life on surfaces. Emerg Infect Dis 8: 881-890

5. Geipel U, Hermann M (2004) Das infizierte Implantat. Teil1. Bakteriologie. Orthopäde 33: 1411-1426

6. Gristina AG (1987) Biomaterial-centered infection: microbial adhesion versus tissue integration. Science 237: 1588-1595

7. Gustilo RB, Anderson JT (1976) Prevention of infection in the treatment of one thousand and twenty-five open fractures of long bones: retrospective and prospective analyses. J Bone Joint Surg Am 58: 453-458

8. Hofmann GO, Bar T, Bühren V (1997) Osteosyntheseimplantat und früher postoperativer Infekt: Sanierung mit oder ohne Materialentfernung? Chirurg 68: 1175-1180

9. McGraw JM, Lim EV (1988) Treatment of open tibial-shaft fractures: external fixation and secondary intramedullary nailing. J Bone Joint Surg Am 70: 900-911

10. Patel R (2005) Biofilms and antimicrobial resistance. Clin Orthop 437: 41-47

11. Smektala R, Wenning M, Ekkernkamp A (1999) Schenkelhalsfraktur: Analyse der Ergebnisse externer Qualitätssicherung - Ein Bericht über 22.556 Patienten. Chirurg 70: 1330-1339

12. Stewart PS, Costerton JW (2001) Antibiotic resistance of bacterias in biofilms. Lancet 358: 135138

13. Tsukayama DT, Estrada R, Gustillo RB (1997) Infection after total hip arthroplasty. A study of the treatment of one hundred and six infections. J Bone Joint Surg Am 78: 512-523

14. Trampuz A, Zimmerli W (2005) Prosthetic joint infections: update in diagnosis and treatment. Swiss Med Wkly 135: 243-251

15. Zimmerli W, Waldvogel FA, Vaudaux P et al. (1982) Pathogenesis of foreign body infections: description and characteristics of an animal model. J Infect Dis 146: 487-497

16. Zimmerli W, Trampuz A, Ochsner PE (2004) Prosthetic joint infections. N Engl J Med 351: 16451654 